Laverde-Sarmiento, M., Lezama-Palomino. J., García-Carrillo, J., Montoya-Valencia, I. y Pérez-Castro, E. (2019). El efecto en el costo de capital de las principales empresas colombianas listadas en el índice bursátil COLCAP, tras la adopción de las Normas Internacionales de Información Financiera. Contaduría Universidad de Antioquia, 74, 153-.170 Doi: https://doi.org/10.17533/udea.rc.n74a07

\title{
El efecto en el costo de capital de las principales empresas colombianas listadas en el índice bursátil COLCAP, tras la adopción de las Normas Internacionales de Información Financiera
}

Miguel-Ángel Laverde-Sarmiento miguellaverde@usantotomas.edu.co Universidad Santo Tomás

Juan-Carlos Lezama-Palomino juanlezama@usantotomas.edu.co Universidad Santo Tomás

Jorge-Fernando García-Carrillo jorge.garciac@usantotomas.edu.co Universidad Santo Tomás

Iván-Camilo Montoya-Valencia ivanmontoya@usantotomas.edu.co Universidad Santo Tomás

Edgar-Alejandro Pérez-Castro edgarperezc@usantotomas.edu.co Universidad Santo Tomás 
El efecto en el costo de capital de las principales empresas colombianas listadas en el índice bursátil COLCAP, tras la adopción de las Normas Internacionales de Información Financiera

Resumen: El costo de capital se entiende como la rentabilidad esperada sobre la inversión realizada en la empresa por parte de los inversionistas (Besley, Brigham, y Gomez, 2001), este indicador se puede ver afectado por el cambio de estándares contables. Es por esto que el objetivo de la investigación es identificar el efecto en el costo de capital de las principales empresas colombianas listadas en el índice bursátil COLCAP, tras la adopción de las Normas Internacionales de Información Financiera (IFRS), por medio del análisis de diferentes empresas entre el 2009 y 2017, a través de los modelos Mínimos Cuadrados Generalizados Factibles (FGLS) y con Error Estándar Corregido en el Panel (PCSE), tomando información calculada bajo el estándar contable local y el internacional. Se evidencia que el implementar las IFRS, no genera un efecto significativo en el costo de capital, a diferencia de variables como el endeudamiento, el margen operacional, el ROE y el crecimiento de las empresas. Igualmente, se concluye que a nivel general la normativa contable inicial y la posterior son indiferentes para el cálculo del costo de capital, sin embargo, a nivel particular esta implementación hizo que algunas variables específicas si tuvieran efecto.

Palabras claves: Costo de Capital, estructura financiera, CAPM, Datos panel, FGLS, PCSE.

The effect on the capital cost of the main Colombian companies listed on the COLCAP stock index after the adoption of International Financial Reporting Standards

Abstract: Capital cost is understood as the expected returns on investments made in the company by investors (Besley, Brigham, y Gomez, 2001). This indicator can be affected by the change in accounting standards. The aim of this research is to identify the effect on the capital cost of the main Colombian companies listed on the COLCAP stock index after the adoption of International Financial Reporting Standards (IFRS). This is done by analyzing different companies between 2009 and 2017 through Feasible Generalized Least Squares (FGLS) and Panel-Corrected Standard Error (PCSE), with information calculated under local and international accounting standards. It is evidenced that implementing IFRS does not generate a significant effect on capital cost, unlike variables such as indebtedness, operating margin, ROE and company growth. Similarly, it is concluded that at general level, both the initial and subsequent accounting standards are indifferent for calculating capital cost; however, at particular level, this implementation caused some specific variables to have effect.

Keywords: Capital cost, financial structure, CAPM, panel data, FGLS, PCSE.

\section{L'impact de l'adoption des Normes Internationales d'Information Financière sur les entreprises colombiennes figurant dans l'indice boursier COLCAP}

Résumé : Le coût du capital est entendu comme la rentabilité attendue sur l'investissement fait par les investisseurs dans l'entreprise (Besley, Brigham y Gómez, 2001). Cet indicateur peut être affecté par le changement des normes comptables. Ainsi, le but de cette étude est d'identifier l'effet sur le coût des capitaux des principales intreprises colombiennes figurant dans l'indice boursier COLCAP après l'adoption des Normes Internationales d'Information Financière (IFRS), grâce à l'analyse faite sur plusieurs entreprises entre 2009 et 2017, au moyen de la méthode des moindres carrés généralisés factibles (MCGF) accompagnée des erreurs-type corrigés par panel (PCSE), en prennant l'information calculée sous les normes comptable nationale et internationale. On constate que la mise en place des IFRS n'a pas un impact important sur le coût du capital, contrairement à des variables telles que l'endettement, la marge d'exploitation, le return on equity (ROE) et la croissance des entreprises. De même, il est conclu que, de facón générale, la norme comptable utilisée précédement est différente de la postérieure en ce qui concerne le calcul du coût du capital. Néanmoins, de façon particulière, cette mise en ouvre a fait que certains variables spécifiques aient de l'effet.

Mots-clés: coût du capital, structure financière, données de panel, MEDAF, MCGF, PCSE.

O Efeito no Custo de Capital das principais empresas colombianas listadas no Índice da Bolsa de Valores COLCAP, após a adoção das Normas Internacionais de Informação Financeira.

Resumo: Entende-se o Custo de Capital como a rentabilidade esperada sobre a inversão realizada na empresa por parte dos inversionistas (Besley, Brigham y Gomez, 2001), este indicador pode-se ver alterado pela mudança de padrões contábeis. É por isso que o objetivo desta pesquisa é identificar o efeito no Custo de Capital das principais empresas colombianas listadas no Índice da Bolsa de Valores COLCAP, após a adoção das Normas Internacionais de Informação Financeira (IFRS), mediante a análise de diversas empresas entre o ano 2009 e o ano 2017, através dos Métodos de Mínimos Quadrados Generalizados Factíveis (FGLS) e com o Desvio Padrão Corrigido no Painel (PCSE), pegando informação calculada sob o padrão contábil local e o internacional. Evidencia-se que a implementação das IFRS, não ocasiona um efeito significativo no Custo de Capital, diferentemente das variáveis como o endividamento, margem operacional, o ROE e o crescimento das empresas. Assim também se conclui que no geral, a normativa contábil inicial e a posterior são indiferentes para o cálculo do Custo de Capital; porém, a um nível particular esta implementação fez que algumas variáveis específicas tivessem efeito.

Palavras chave: Custo de Capital, estrutura financeira, CAPM, Dados Painel, FGLS, PCSE. 
Cont. udea (enero-junio), pp. 153-170. (C) Universidad de Antioquia-2019.

\title{
El efecto en el costo de capital de las principales empresas colombianas listadas en el índice bursátil COLCAP, tras la adopción de las Normas Internacionales de Información Financiera
}

\author{
Miguel-Ángel Laverde-Sarmiento, Juan-Carlos Lezama-Palomino, \\ Jorge-Fernando García-Carrillo, Iván-Camilo Montoya-Valencia y \\ Edgar-Alejandro Pérez-Castro \\ Doi: https://doi.org/10.17533/udea.rc.n74ao7
}

Primera versión recibida en abril de 2019 - Versión final aceptada en junio de 2019

\section{Introducción}

$\mathrm{L}$ as Normas Internacionales de Información Financiera (NIIF-IFRS) se han implementado a lo largo del tiempo en 120 países (American Institute of Certified Public Accountants, 2019). En Colombia este proceso se ha realizado en lo corrido de la última década, lo cual ha generado retos en la elaboración y en el análisis de la información contable y financiera; esto lo ratifican Byard, Li, y Yu (2011) al afirmar que al cambiar a un estándar contable internacional se evidencia una evolución de las herramientas de decisión que puede traer modificaciones, específicamente a los estados financieros.

Aunque con las IFRS mejora la información financiera en temas de presentación, transparencia y comparabilidad (Bae, Tan, y Welker, 2008), es importante tener presente otras afectaciones de la aplicación de este estándar, debido a que puede reducir la calidad de los informes financieros, generar una volatilidad en los resultados o una disminución en la fiabilidad de la información para la toma de decisiones (Ball, 2006); por tal motivo, es necesario indagar diferentes variables que puedan ser afectadas.

Una de estas variables puede ser la del costo de capital, la cual hace referencia a la tasa de retorno requerida de la inversión de una entidad que refleja el valor de los instrumentos a largo plazo (Munteanu L. , 2011). Adicionalmente, este indicador es importante, debido a que pueden determinar el costo de oportunidad del capital de las empresas y los inversionistas (Valderrama, Díez, y Gaitán, 2011), de este modo se debe tener en cuenta que 
Laverde, M., Lezama, J., García, J., Montoya, I., Pérez, E. El efecto en el costo de capital...

este indicador puede verse afectado por diferentes variables como, la tasa de interés, el salario, el precio de bienes de capital, la depreciación, los impuestos, los subsidios y los aranceles (Valderrama, Díez, y Gaitán, 2011).

Dentro de las principales herramientas utilizadas para calcular esta variables, se encuentra el modelo CAPM (Capital Asset Pricing Model) el cual tiene un gran desarrollo a lo largo de la historia a través de los postulados de Markowitz (1952), Sharpe (1964), Litner (1965), Mossin (1966) y Jensen, Black, y Scholes (1972), el cual se calcula teniendo en cuenta el enfoque de mercados en estado de competencia perfecta y se asume una relación directa entre el costo y el riesgo de la inversión.

Debido a lo anterior, esta investigación pretende analizar el efecto que tiene la implementación de las IFRS en el costo del capital de las principales empresas del índice bursátil COLCAP en Colombia. Para cumplir este propósito, inicialmente se realiza una revisión literaria sobre los referentes teóricos y los trabajos que se han realizado con un enfoque similar. A partir de esta revisión literaria se selecciona un modelo para medir el efecto de las IFRS sobre el costo del capital en las principales empresas que conforman el índice COLCAP desde los años 2009 al 2017, para esto seidentifican algunas variables financieras que afectan el costo de capital, luego, a través del modelo CAPM se estima el valor de la variable dependiente. Posteriormente, se ejecuta el modelo y se hacen unas pruebas de especificidad y, por último, se determinan los resultados y conclusiones del trabajo.

\section{Revisión literaria}

El objetivo de la teoría financiera relacionada con el costo de capital es la de identificar una estructura óptima de financiación propia para las entidades, esta se divideen tres grandes enfoques: el primero es el teorema de ModiglianiMiller (1958) en el cual identifica que el valor del capital de una entidad en un mercado determinado, suponiendo una ausencia de costos de transacción de liquidación, impuestos y asimetrías de información, le es indiferente financiarse por medio de terceros o de recursos propios (Tenjo, López, yZamudio, 2006).

El segundo hace referencia a la teoría del portafolio, la cual se enfoca en elaborar una metodología para la selección de un portafolio de inversión, teniendo en cuenta valores futuros esperados, la maximización del retorno y la minimización de los riesgos (Markowitz, 1952) y por último el enfoque del mercado de capitales, el cual estudia la valoración de las inversiones y la determinación de los precios, lo cual es la base teórica del modelo de valoración de activos financieros (de ahora en adelante CAPM por sus siglas en inglés) (Lucas, 1984) estos enfoques evidencian la importancia que le dan diferentes autores, a la identificación de las variables que pueden afectar la rentabilidad de una inversión, como en el caso de este estudio, el cual pretende 
identificar, la implicación de la implementación de un nuevo marco normativo contable.

Una de estas variables es la del Costo de Capital la cual está definida como la rentabilidad promedio que los capitalistas esperan sobre su inversión en una empresa específica, es decir, el valor de los fondos que debe ofrecer una entidad para atraer inversionistas (Besley, Brigham, y Gomez, 2001), Weston (1963) lo define como el porcentaje de descuento que se otorga a una inversión y Weston, Brigham, y Mont (1987) mencionan que para realizar factores de producción se requiere de un costo específico, que en términos de inversión se le conoce como costo de capital.

En este estudio la variable dependiente es la anteriormente mencionada, la cual se calcula tomando como referencia el modelo CAPM, el cual se utiliza para estimar el retorno esperado de una inversión, teniendo en cuenta un riesgo determinado, este modelo está referenciado por Gordon y Shapiro (1956), Gordon (1997), Ohlson-Juettner (2005), y Brealey, S., y F (2010), este modelo es el más utilizado, y es el de más fácil aplicación, sin embargo presenta limitaciones, como lo es la estimación de la tasa libre de riesgo, el cálculo del beta y en algunos casos la determinación de la variable proxy de otros mercados para determinar la prima de riesgo (Botosan C. A., 2006).

Por otro lado Farfán (2010) menciona que la información contable a inicio de los años setenta, pasó de tener un enfoque patrimonial para los inversionistas, a uno relacionado con el paradigma de la utilidad de información, el cual se enfoca en la toma de decisiones a múltiples usuarios. Esto está fundamentado en el enfoque pragmático de Hendriksen y Fernandez (1981) en el cual se observa que la utilidad es la base para una interpretación correcta de la información contable; por otro lado Belkaoui (1993) señala que la contabilidad debe cumplir con el paradigma de la utilidad de la decisión, la cual señala que los datos contables deben tener capacidad de predicción y esto los hace útiles para la toma de decisiones. Es así que el objetivo de la información financiera ha sido el de buscar reducir la asimetría de la información de los diferentes usuarios.

Adicionalmente Munteanu (2011), Lambert, Leuz, y Verrechia (2007), Foster (2013), Botosan (1997), Diamond y Verrecchia (1991) y Urquiza, Navarro, y Trombetta (2012) están de acuerdo en que, al aumentar la propagación de la información, se reduce el costo de capital, debido a que aumenta la confianza por parte de los inversionistas, disminuye los costos de transacción y aumenta el intereses de otros posibles inversores. Adicionalmente estos factores disminuyen la asimetría de la información y refuerzan el paradigma de la utilidad contable. Por lo tanto, este trabajo busca identificar si la aplicación de un nuevo marco contable afecta en el costo de capital y de esta forma afectar la utilidad y la asimetría de la información contable 
Laverde, M., Lezama, J., García, J., Montoya, I., Pérez, E. El efecto en el costo de capital...

Asimismo, con la finalidad de tener un marco de referencia, en la tabla 1 se muestran los diferentes estudios similares a esta investigación, en los cuales se puede apreciar, que la mayoría, identifican una reducción del costo de capital al aplicar las IFRS. En relación con los países europeos existe una cantidad considerable de investigaciones, que analizan el efecto en el costo de capital y el resultado depende no sólo del tipo de empresa objeto de estudio, sino que además de factores externos que se relacionan con el riesgo. En cambio, en investigaciones de países latinoamericanos no son abundantes y se han desarrollado principalmente en Brasil. En esta revisión no se evidenció trabajos similares en Colombia por lo cual hace que el desarrollo de este documento sea una contribución empírica a los trabajos que se han realizado en el mundo.

Tabla 1: Síntesis del estado del arte

\begin{tabular}{|c|c|c|c|c|c|}
\hline Autor & Año & Región & $\begin{array}{l}\text { Periodo de } \\
\text { estudio }\end{array}$ & $\begin{array}{l}\text { Modelo de } \\
\text { medición del } \\
\text { efecto }\end{array}$ & Conclusiones \\
\hline $\begin{array}{l}\text { Góis, de Lima, de } \\
\text { Sousa, y Malacrida }\end{array}$ & $(2018)$ & $\begin{array}{l}31 \text { Países al } \\
\text { rededor del } \\
\text { mundo }\end{array}$ & $2002-2007$ & $\begin{array}{l}\text { Mínimos } \\
\text { cuadrados } \\
\text { ordinarios }\end{array}$ & Reduccióndel costodecapital \\
\hline Persakis y Latridis & $(2017)$ & $\begin{array}{l}\text { Zona Euro y } \\
\text { países Asiáticos }\end{array}$ & 2000-2014 & $\begin{array}{l}\text { Regresión } \\
\text { lineal }\end{array}$ & $\begin{array}{l}\text { Se encontró una asociación } \\
\text { negativa }\end{array}$ \\
\hline Agustin & $(2016)$ & $\begin{array}{l}31 \text { Europa } \\
\text { América y Asia }\end{array}$ & 2007-2011 & $\begin{array}{l}\text { Regresión de } \\
\text { datos panel }\end{array}$ & $\begin{array}{l}\text { No hay evidencia que exista un } \\
\text { impacto positivo }\end{array}$ \\
\hline $\begin{array}{l}\text { Houqe, Monem, } \\
\text { y Zijl }\end{array}$ & $(2016)$ & Nueza Zelanda & $2003-2008$ & Regresión & Reduccióndelcostodecapital \\
\hline $\begin{array}{l}\text { Silva, Augusto, y } \\
\text { Costa }\end{array}$ & $(2016)$ & Brasil & 1998-2004 & $\begin{array}{l}\text { Regresión con } \\
\text { datos panel }\end{array}$ & $\begin{array}{l}\text { No afecta estadísticamente al } \\
\text { costo de capital }\end{array}$ \\
\hline $\begin{array}{l}\text { Gatsios, Da Silva, } \\
\text { Ambrozini, Neto, } \\
\text { y Lima }\end{array}$ & $(2016)$ & Brasil & 2004-2013 & $\begin{array}{l}\text { Diferencias en } \\
\text { diferencias }\end{array}$ & Reducción del costo de capital \\
\hline Kim, Shi, y Zhou, & $(2014)$ & $\begin{array}{l}34 \text { Países al } \\
\text { rededor del } \\
\text { mundo }\end{array}$ & 1998-2004 & Regresión & $\begin{array}{l}\text { Reducción del costo de } \\
\text { capital principalmente en las } \\
\text { que tienen una estructura } \\
\text { económica débil. }\end{array}$ \\
\hline $\begin{array}{l}\text { Grabinski, Kedzior, } \\
\text { y Krasodomska }\end{array}$ & $(2014)$ & Polonia & 2002-2013 & $\begin{array}{l}\text { Análisis } \\
\text { estadístico }\end{array}$ & Reducción del costo de capital \\
\hline $\begin{array}{l}\text { lonașcu, lonaşcu, } \\
\text { Sâcârin, y Minu, }\end{array}$ & $(2014)$ & Rumania & 1990-2014 & $\begin{array}{l}\text { Análisis } \\
\text { estadístico }\end{array}$ & Reducción del costo de capital \\
\hline $\mathrm{Li}$ & $(2010)$ & Unión Europea & 1995-2006 & $\begin{array}{l}\text { Diferencias en } \\
\text { diferencias }\end{array}$ & $\begin{array}{l}\text { Reducción del costo de capital, } \\
\text { depende fuerte marco legal }\end{array}$ \\
\hline $\begin{array}{l}\text { Daske, Hail, Leuz, } \\
\text { y Verdi }\end{array}$ & $(2008)$ & $\begin{array}{l}26 \text { países } \\
\text { alrededor del } \\
\text { mundo }\end{array}$ & 2001-2005 & Datos Panel & $\begin{array}{l}\text { Reducción del costo de capital } \\
\text { antes de la adopción }\end{array}$ \\
\hline $\begin{array}{l}\text { Lee, Walker, y } \\
\text { Christensen }\end{array}$ & $(2008)$ & Europa & $1995-2006$ & $\begin{array}{l}\text { Regresión } \\
\text { Lineal }\end{array}$ & $\begin{array}{l}\text { No afecta estadísticamente al } \\
\text { costo de capital }\end{array}$ \\
\hline
\end{tabular}

Fuente: Elaboración propia 


\section{Metodología}

Para medir el efecto de las IFRS sobre el costo del capital se plantea la siguiente hipótesis de investigación:

$H_{\mathrm{o}} \mathrm{La}$ adopción de las IFRS afectó el Costo esperado de capital de las principales empresas del índice COLCAP

Para dar respuesta a esta hipótesis, se determinó utilizar las primeras nueve empresas con mayor participación en el índice COLCAP a 31 de diciembre de $2017^{1}$, debido a que son las empresas más grandes y representativas de la bolsa de valores colombiana, al poseer el $80 \%$ del comportamiento del mercado. $\mathrm{Al}$ ser un mercado bursátil pequeño el número de empresas son limitadas, por lo cual este trabajo no es representativo para la economía, pero si son empresas de referencia para el mercado de valores en la cual está orientada las IFRS (2018). En la Tabla 2 se señala las empresas objeto de investigación.

Tabla 2: empresas objeto de estudio que pertenecen al índice COLCAP

\begin{tabular}{llc}
\hline Empresa & Acciones en el índice Colcap & Participación \\
\hline Bancolombia & PFBCOLOM, BCOLOMBIA & $18,8 \%$ \\
Grupo Sura & GRUPOSURA, PFGRUPSURA & $12,8 \%$ \\
Grupo Argos & GRUPOARGOS, PFARGOS & $10,0 \%$ \\
Ecopetrol & ECOPETROL & $9,0 \%$ \\
Grupo Nutresa & NUTRESA & $6,9 \%$ \\
Cementos Argos & CEMARGOS, PFCEMARGOS & $6,9 \%$ \\
Grupo Isa & ISA & $6,32 \%$ \\
Grupo Aval & GRUPOAVAL, PFAVAL & $6,1 \%$ \\
Grupo Éxito & ÉXITO & $3,3 \%$ \\
Otros & & $20,0 \%$ \\
\hline
\end{tabular}

Fuente: Elaboración propia con datos de la Bolsa de Valores de Colombia (BVC).

Para el desarrollo de esta investigación se utilizaron fuentes deinformación públicas, en el caso de las empresas se consultaron los estados financieros trimestrales publicados en el portal de la Superintendencia Financiera de Colombia desde el año 2009 al 2017. También se consultaron los precios de cierre de las acciones, el primer y último día del trimestre en el portal de la Bolsa de Valores de Colombia.

Una de las variables a considerar en el estudio, es la del costo de capital, el cual se define como el retorno esperado de un inversionista que invierte en la empresa dado un nivel de riesgo es un valor no observable. Para calcularlo se

1 En esta investigación se excluyó la empresa Grupo Energía Bogotá ya que, al ser una entidad pública, la aplicación de las normas internacionales fue diferente al sector privado, empezando que las normas que aplicaron fueron las IPSAS lo que afecta considerablemente la valoración de los estados financieros. 
Laverde, M., Lezama, J., García, J., Montoya, I., Pérez, E. El efecto en el costo de capital...

han propuesto diferentes metodologías; ${ }^{2}$ sin embargo, una de las formas más utilizadas para estimarlo y que se utiliza en este trabajo es el modelo CAPM (Brealey, S., y F., 2010). El cual se calcula de la siguiente manera:

Donde:

$$
k e=r_{f}+\beta\left(r_{m}-r_{f}\right)
$$

ke: es el costo de capital de la empresa

$r_{f}$ : es la tasa libre de riesgo

$\beta$ : es la beta apalancado de la empresa

$\left(r_{m}-r_{f}\right)$ : es la prima por riesgo de mercado

Este modelo es usado para calcular una rentabilidad esperada, al realizarlo con datos históricos pierde este propósito, aunque sigue siendo un buen estimador del costo del capital pues se está utilizando datos reales y no proyectados. Además, se debe tener en cuenta que el CAPM se debe ajustar a países en desarrollo, pues los mercados no son perfectos, además existe poca información o pocas empresas, esto hace que las betas no sean significativas (Silva, Augusto, y Costa, 2016); para resolver estas limitación se usan betas equivalentes del sector de la empresa de otros países como los de Estados Unidos o los de países emergentes similares (Silva, Augusto, y Costa, 2016).

En este caso, para estimar las betas de cada empresa, dado sus limitaciones para el cálculo, se utilizó como proxy las betas desapalancadas (unlevered) por sector de los países emergentes y se incluye el apalancamiento de las empresas objeto de estudio. Para este propósito, se consultó las betas de países emergentes de las bases de Damodaran ${ }^{3}$ para los periodos 2009 al 20174, las cuales son actualizadas al principio de año, pues esta información se derivada de 102 naciones emergentes y más de 21000 empresas, incluida Colombia y las empresas de estudio ${ }^{5}$,

2 Entre los más conocidos está el modelo de crecimiento de Gordon (Gordon y Shapiro, 1956), (Gordon J., 1997), la ratio PEG (Easton, 2004) y el modelo de Modelo Ohlson-Juettner y sus variantes.

3 Dada la ausencia de información pública, Damodaran calcula información del mercado con fines académicos (Damodaran, s.f.)

4 Se utilizó las betas de Estados Unidos en los años 2009 al 2010, pues la información disponible de las betas de países emergentes es a partir del año 2011

5 Más información sobre las empresas y países que se basa el cálculo se puede consultar en el siguiente enlace: http://www.stern.nyu.edu/ adamodar/pc/datasets/indname.xls 
Tabla 3: Betas Desapalancadas del sector de las empresas de estudio

\begin{tabular}{lllllllllll}
\hline \multicolumn{1}{c}{ Empresa } & \multicolumn{1}{c}{ Sector* $^{*}$} & 2009 & 2010 & 2011 & 2012 & 2013 & 2014 & 2015 & 2016 & 2017 \\
\hline Aval & Bank (Money Center) & 0,28 & 0,30 & 0,36 & 0,46 & 0,46 & 0,45 & 0,39 & 0,30 & 0,40 \\
Bcolombia & Bank (Money Center) & 0,28 & 0,30 & 0,36 & 0,46 & 0,46 & 0,45 & 0,39 & 0,30 & 0,40 \\
Cem_argos & Construction Supplies & 0,84 & 0,83 & 0,51 & 0,74 & 0,60 & 0,74 & 0,78 & 0,72 & 0,92 \\
Ecopetrol & Oil/Gas (Integrated) & 0,56 & 0,60 & 0,71 & 1,01 & 0,92 & 1,02 & 1,05 & 0,79 & 0,86 \\
EEB & Power & 0,63 & 0,65 & 0,34 & 0,52 & 0,50 & 0,49 & 0,53 & 0,46 & 0,57 \\
Éxito & Retail (General) & 1,13 & 1,21 & 0,56 & 0,79 & 0,80 & 0,70 & 0,95 & 0,86 & 1,03 \\
Grupoargos & Construction Supplies & 0,84 & 0,83 & 0,51 & 0,74 & 0,60 & 0,74 & 0,78 & 0,72 & 0,92 \\
Isa & Power & 0,63 & 0,65 & 0,34 & 0,52 & 0,50 & 0,49 & 0,53 & 0,46 & 0,57 \\
Nutresa & Food Processing & 0,69 & 0,72 & 0,47 & 0,71 & 0,62 & 0,58 & 0,70 & 0,62 & 0,71 \\
Sura & Diversified & 0,57 & 0,68 & 0,48 & 0,64 & 0,53 & 0,65 & 0,52 & 0,40 & 0,48 \\
\hline
\end{tabular}

*Para que la información sea consistente, la clasificación por sector es la misma que usa Damodaran para las empresas colombianas. Fuente: Elaboración propia con datos obtenido de Damodaran Online(s.f.)

Una vez obtenido las betas desapalancadas del sector se usa la siguiente ecuación para determinar la beta apalancada de cada empresa por trimestre (Damodaran, 2002):

Donde:

$$
\beta_{e}=\beta_{\operatorname{proxy}}\left[1+\frac{D \cdot(1-t)}{E}\right]
$$

Bproxy: es la beta desapalancada del sector

$\frac{D \cdot(1-t)}{E}$ : es relación deuda sin el efecto del impuesto sobre patrimonio de la empresa $^{6}$.

$t:$ es la tasa de impuesto.

Para determinar la tasa libre de riesgo, se utilizaron los Títulos de Tesorería (TES) que estaban vigentes en el período de estudio, la información fue consultada en el portal financiero del grupo AVAL, y para estimarlo se calculó el promedio trimestral dela rentabilidad de los TES con vencimiento a junio de 2016 , octubre de 2018, julio de 2020, mayo de 2022, julio de 2024 y agosto de 2026 para los años 2009 al 2017.

Para obtener la prima por riesgo de mercado, se utilizaron los datos calculados por Damodaran para Colombia basado en el rating de Moody para los años 2009 al 2017.

6 La información de la Deuda, Patrimonio y la tasa de impuesto se obtuvo de los estados financieros de las empresas. Para la tasa de impuesto se estimó de acuerdo con la provisión del impuesto de renta de cada empresa. 
Laverde, M., Lezama, J., García, J., Montoya, I., Pérez, E. El efecto en el costo de capital...

Tabla 4: Prima por riesgo de mercado

\begin{tabular}{cc}
\hline Año & Prima de Riesgo \\
\hline 2009 & $7,5 \%$ \\
2010 & $8,0 \%$ \\
2011 & $9,0 \%$ \\
2012 & $8,8 \%$ \\
2013 & $8,3 \%$ \\
2014 & $8,6 \%$ \\
2015 & $9,2 \%$ \\
2016 & $8,4 \%$ \\
2017 & $7,3 \%$ \\
\hline
\end{tabular}

Fuente:Elaboración propia con datos obtenidodeDamodaran Online.

Una vez calculado el costo de capital y en línea a las investigaciones relacionadas que se ha realizado en los diferentes países (Castillo, Menédez, y Orgaz, 2014, Gatsios, Da Silva, Ambrozini, Neto, y Lima, 2016, Houqe, Monem, y Zijl, 2016, Li, 2010, Silva, Augusto, y Costa, 2016) se desarrolla el siguiente modelo en el que se añade una variable dummy para medir el efecto en la adopción de las IFRS:

$$
\begin{aligned}
& \text { ke }_{i t}=\beta_{0}+\beta_{1} D_{-} \text {IFRS }_{i t}+\beta_{2} \text { Inflación }_{i t}+\beta_{3} \text { Endeudamiento }_{i t}+\beta_{4} \text { ROE }_{i t}+ \\
& \beta_{5} \text { MO }_{i t}+\beta_{6} \text { TamEmpresa }_{i t}+\beta_{7} \text { Crecimiento }_{i t}+\beta_{8} \text { Retorno }_{i t}+\beta_{9} \text { DJI I I }_{i t}+u_{i t}
\end{aligned}
$$

Donde:

$k e_{i t}$ : es el costo de capital de cada empresa $i$ para el trimestre $t$.

$D_{\_} I F R S_{i t}$ : es la variable dummy que toma el valor de $\mathrm{o}$ antes de la adopción de las IFRS y 1 después de la adopción de las IFRS de cada empresa $i$ para el trimestre $t$.

Inflación $n_{i t}$ : es la tasa de inflación anual calculada por el DANE para el trimestre t basado en $\mathrm{Li}$ (2010).

Endeudamiento $_{i t}$ : es el nivel de endeudamiento calculado como pasivos sobre activos de cada empresa $i$ para el trimestre $t$ basado en y Li(2010), Castillo et al. (2014) y Silva et al. (2016).

$R O E_{i t}$ : es el retorno sobre patrimonio calculado como la utilidad neta acumulada sobre patrimonio de cada empresa $i$ para el trimestre $t$ basado en Castillo y et. al. (2014) y Silva et al. (2016).

$M O_{i t}$ : es el margen operacional calculado como la utilidad operacional acumulada sobre ventas o ingreso netos acumulado de cada empresa i para el trimestre t basado en Silva et al. (2016).

TamEmpresa $_{i t}$ : mide el tamaño de la empresa usando el logaritmo natural del activo de cada empresa $i$ para el trimestre $t$ basado en Houqey et. al. (2016). 
Crecimiento $_{i t}$ : es la relación patrimonio sobre capitalización bursátil, para calcular el capital bursátil se tomó el precio de la acción y se multiplicó por el número de acciones diferenciando acciones ordinarias y preferenciales de cada empresa $i$ para el trimestre $t$ basado en Houqe et. al. (2016)

Retorno $_{i t}$ : es el retorno de la acción calculado como $\operatorname{Ln}\left(\frac{\text { Precio }_{t}}{\text { Precio }_{t-1}}\right)$ de cada empresa para el trimestre basado en Silva et al. (2016).

$D J I_{i t}:$ es el retorno del índice Dow Jones Industrial Average calculado como Ln $\left(\frac{\text { Preciot }_{\text {Precio }}}{\text { pr-1 }}\right)$ que se usa como variable proxy para medir el efecto de los mercados internacionales sobre el capital social basado en Li (2010) y Castillo et. al. (2014)

Para la estimación del modelo se realiza el test del multiplicador de Langrage de Breusch-Pagan (1980) para determinar si es un modelo de regresión agrupada o de datos panel. Si el modelo es de datos panel se usará el test de Hausman (1978) y determinar si es válido realizarlo con efectos aleatorios o efectos fijos. También se realizan pruebas estadísticas para garantizar su consistenciay especificar bien el modelo. Es importante aclarar que gran parte de la metodología se tomó como referencia el trabajo de Castillo, Menédez, y Orgaz (2014), de igual forma se menciona que existen más variables que no se incluyeron en el estudio, que pueden afectar al costo de capital, las cuales pueden ser analizadas en investigaciones posteriores.

\section{Resultados}

Se aprecia al usar el modelo FGLS que las variables que afectan positivamente el costo de capital son la constante, la inflación y el endeudamiento. Estos resultados se dan debido a que si aumenta la inflación los inversionistas esperan una mayor rentabilidad y en cuanto al endeudamiento, el efecto de apalancamiento logra que aumente el riesgo de la inversión y a su vez la rentabilidad. Mientras que las que afectan negativamente son el ROE, el Margen Operativo (MO) y el crecimiento, debido a que su aumento, es un buen indicador del desempeño de la empresa, esto genera una mayor seguridad en la inversión, hace que disminuya el riesgo y de igual forma el costo de capital.

En ambos modelos FGLS y PCSE la variable dummy no es significativa, por lo tanto, se rechaza la hipótesis nula que la adopción de las IFRS afectó el costo esperado de capital de las principales empresas del índice COLCAP en concordancia con Lee et. al. (2008) y Gatsios, Da Silva (2016). De acuerdo con Gatsios et al (2016) la no significancia de la variable dummy puede obedecerse a dos aspectos: Primero, se puede observar en la dificultad de medir el costo de capital, ya que es una variable que no es directamente observada y su medición obedece a valores subjetivos; y segundo, a la especificación del modelo, ya que este deja de considerar el efecto posible de las diferentes políticas contables de cada empresa ya que la toma como un todo. 
Laverde, M., Lezama, J., García, J., Montoya, I., Pérez, E. El efecto en el costo de capital...

Este resultado contribuye al debate académico sobre la relación entre la calidad de la información al adoptar las IFRS y su efecto en el costo del capital. La ausencia del impacto de la adopción de las IFRS puede explicarse por la fragilidad institucional, el bajo poder de aplicación para la correcta implementación de las normas internacionales, la alta influencia hacia normas tributarias y el poco desarrollo del mercado de capitales en el país (Gatsios et al., 2016).

Sin embargo, el efecto de las IFRS puede aparecer a medida que pasa el tiempoylas normas tengan mayor fuerza, lo que permite quelas empresas ylos accionistas se vayan adaptando a los cambios y sea más efectivo la mejora de la calidad de la información contable (Gatsios et al., 2016).

Después de analizar los resultados más representativos, a continuación, se detalla el proceso dela aplicación y ajustes de los modelos econométricos. Para las nueve empresas de estudio se calcularon cada una de las variables desde el primer trimestre de 2009 al cuarto trimestre de 2017, en el cual se obtuvo 324 observaciones. En la Tabla 5 se hace una descripción de las variables del modelo.

Tabla 5: Descripción de las variables del modelo

\begin{tabular}{llccccc}
\hline \multicolumn{1}{c}{ Variable } & \multicolumn{1}{c}{ Descripción } & Obs. & Media & Desviación Estándar & Mínimo & Máximo \\
\hline KE & Costo de Capital & 324 & $16,34 \%$ & $5,06 \%$ & $8,99 \%$ & $34,60 \%$ \\
D_IFRS & Dummy Impacto IFRS & 324 & 0,33 & 0,47 & 0 & 1 \\
Inflación & Inflación & 324 & $3,84 \%$ & $1,72 \%$ & $1,84 \%$ & $8,60 \%$ \\
Endeudamiento & Endeudamiento & 324 & $39,88 \%$ & $24,60 \%$ & $1,06 \%$ & $90,64 \%$ \\
ROE & Retorno sobre Patrimonio & 324 & $6,60 \%$ & $6,02 \%$ & $-17,59 \%$ & $27,70 \%$ \\
MO & Margen Operacional & 324 & $40,08 \%$ & $32,00 \%$ & $1,16 \%$ & $98,78 \%$ \\
TamEmpresa & LN Activos & 324 & 30,84 & 0,95 & 29,05 & 32,95 \\
Crecimiento & Crecimiento de la Empresa & 324 & 1,52 & 0,75 & 0,58 & 4,58 \\
Retorno & Retorno de la acción & 324 & $0,22 \%$ & $1,36 \%$ & $-5,37 \%$ & $6,30 \%$ \\
DJI & Retorno índice DJI & 324 & $0,30 \%$ & $0,70 \%$ & $-1,57 \%$ & $1,54 \%$ \\
\hline
\end{tabular}

Fuente: Elaboración propia.

El primer paso para estimar el modelo es verificar si es preferible usar modelo de regresión agrupada (pooled con $\mathrm{MCO}$ ) o de datos panel. En la tabla 6 se muestra los resultados del test ML de Breusch-Pagan (1980) y se puede apreciar que el valor p es menor al $5 \%$ rechazando la hipótesis nula e indicando que es mejor utilizar un modelo de datos panel. 
Tabla 6: Test ML de Breusch-Pagan

\begin{tabular}{lcc}
\hline & \multicolumn{1}{c}{ Var } & sd=sqrt(Var) \\
\hline$K E$ & 0,002558 & 0,0505767 \\
$e$ & 0,0003896 & 0,0197378 \\
$u$ & 0,0002221 & 0,0149022 \\
\hline Test: & \\
Var $(u)=O$ \\
chibar2 $($ o1 $)=359,1$ \\
Prob $>$ chibar2= $\mathbf{0 , 0 0 0 0}$ \\
\hline
\end{tabular}

Fuente: Elaboración propia.

Para determinar si el modelo de datos panel es mejor con efectos fijos o aleatorios se usa el test de Hausman (1978), tal como se observa en la Tabla 7, el valor p es menor al $5 \%$ y por tanto se rechaza la hipótesis nula, aceptando que es mejor un modelo de datos panel con efectos fijos.

Tabla 7: Test de Hausman

\begin{tabular}{|c|c|c|c|c|}
\hline \multirow{3}{*}{ Variables } & \multicolumn{4}{|c|}{ Coeficientes } \\
\hline & (b) & (B) & $(b-B)$ & sqrt(diag(V_b-V_B)) \\
\hline & Efectos fijos & Efectos aleatorios & Diferencia & $\mathrm{S}, \mathrm{E}$ \\
\hline $\mathrm{D}$ & 0,0000819 & $-0,0086145$ & 0,0086964 & 0 \\
\hline Inflación & 0,1497104 & 0,1883774 & $-0,038667$ & 0 \\
\hline Endeudamiento & 0,1004068 & 0,1242905 & $-0,0238836$ & 0,0035985 \\
\hline ROE & $-0,0462135$ & $-0,0449684$ & $-0,0012451$ & 0 \\
\hline MO & 0,0124596 & 0,0178212 & $-0,0053617$ & 0,0029385 \\
\hline TamEmpresa & $-0,0227206$ & $-0,0095372$ & $-0,0131834$ & 0,0019246 \\
\hline Crecimiento & $-0,0142889$ & $-0,0100376$ & $-0,0042513$ & 0 \\
\hline Retorno & $-0,0785482$ & $-0,0464748$ & $-0,0320734$ & 0 \\
\hline DJI & $-0,0266861$ & $-0,0939736$ & 0,0672875 & 0 \\
\hline \multicolumn{5}{|l|}{ Test } \\
\hline $\begin{array}{l}\operatorname{chi2}(9)=(b-B)^{\prime}[ \\
\operatorname{chi2}(9)=455,21 \\
\text { Prob }>\text { chi2 }=0\end{array}$ & $\begin{array}{l}\left.\left[b-V_{-} B\right)^{\wedge}(-1)\right]( \\
\text { ооо }\end{array}$ & $-B)$ & & \\
\hline
\end{tabular}

Fuente: Elaboración propia.

Para validar la especificidad del modelo se comprueban tres aspectos: la autocorrelación de los residuos, la correlación cruzada o contemporánea y la heterocedasticidad de los datos, el cual, de no presentarse ninguno de estos aspectos indica que el modelo está bien especificado.

Para validar la autocorrelación de los errores en datos panel se usa el test de Wooldridge (2002) el cual tiene como hipótesis nula la no existencia de autocorrelación de primer orden en los residuos. Al realizar esta prueba en el modelo se rechaza la hipótesis nula por lo tanto el modelo presenta autocorrelación. 
Laverde, M., Lezama, J., García, J., Montoya, I., Pérez, E. El efecto en el costo de capital...

Con respecto a la correlación contemporánea se realiza el test LM de independencia de Breusch-Pagan o test B-P/LM (1980) que tiene como hipótesis nula la independencia transversal. De acuerdo con los resultados de la Tabla 8 se rechaza la hipótesis nula por lo tanto el modelo también presenta problemas de correlación cruzada.

Tabla 8: Test de B-P/LM

\begin{tabular}{lrrrrrrrrr}
\hline & \multicolumn{1}{c}{ e1 } & e2 & e3 & e4 & e5 & e6 & e7 & e8 & e9 \\
\hline e1 & 1 & & & & & & & & \\
e2 & $-0,6216$ & 1 & & & & & & \\
e3 & $-0,5041$ & 0,7778 & $\mathbf{1}$ & & & & & \\
e4 & $-0,5352$ & 0,7611 & 0,9294 & $\mathbf{1}$ & & & & \\
e5 & 0,6559 & $-0,0908$ & $-0,0186$ & $-0,147$ & $\mathbf{1}$ & & & \\
e6 & $-0,2739$ & 0,7456 & 0,9157 & 0,8545 & 0,2127 & 1 & & \\
e7 & 0,5753 & $-0,17$ & $-0,0604$ & $-0,1476$ & 0,5804 & 0,1647 & 1 & \\
e8 & 0,0932 & 0,3375 & 0,6209 & 0,5003 & 0,2872 & 0,6463 & 0,4525 & 1 \\
e9 & $-0,6176$ & 0,9722 & 0,7638 & 0,7482 & $-0,0669$ & 0,7466 & $-0,1488$ & 0,34091
\end{tabular}

Breusch-Pagan LM test of independence: $\operatorname{chi2}(36)=398,265$, Prob $=0,0000$

Fuente: Elaboración propia.

Elúltimo aspecto se usó el test de Wald modificado para heterocedasticidad (Greene, 2000) (Baum, 2001), en donde se rechaza la hipótesis nula indicando que el modelo presenta también problemas de heterocedasticidad.

Para solucionar los tres problemas de especificidad se calculó el modelo de datos panel con estimadores de Mínimos Cuadrados Generalizados Factibles (FGLS) y con Error Estándar Corregido en el Panel (PCSE) ajustando la autocorrelación de los residuos. En la Tabla 9 se comparar los resultados delas dos estimaciones.

Tabla 9: Resultados del modelo

\begin{tabular}{lll}
\hline Variable & FGLS & PCSE \\
\hline D_IFRS & $-0,00127525$ & 0,00071578 \\
Inflación & $0,22925684^{*}$ & 0,13022181 \\
Endeudamiento & $0,12745385^{* * *}$ & $0,134402^{* * *}$ \\
ROE & $-0,01310211^{*}$ & $-0,02374589$ \\
MO & $-0,0127927^{* * *}$ & $-0,0184894^{* *}$ \\
TamEmpresa & $-0,0001819$ & 0,00179077 \\
Crecimiento & $-0,00308388^{*}$ & $-0,00136988$ \\
Retorno & 0,0115382 & $-0,01401272$ \\
DJI & $-0,06818083$ & $-0,01694393$ \\
Constante & $0,1177043^{*}$ & 0,0595536 \\
\hline Nota: ${ }^{*} \mathrm{p}<0.05 ;{ }^{* *} \mathrm{p}<0.01 ;{ }^{* * *} \mathrm{p}<0.001$ & \\
\hline
\end{tabular}

Fuente: Elaboración propia. 
Se aprecia al usar el modelo FGLS que las variables que afectan positivamente el costo de capital social son la constante, la Inflación y el endeudamiento, mientras que los que afecta negativamente son el ROE, Margen Operativo (MO) y el crecimiento. Por otro lado, al utilizar el modelo PCSE solo el Endeudamiento y el MO son significativos.

\section{Conclusiones}

Con la ley 1314 de 2009 el gobierno colombiano promovió la implementación de un sistema contable actualizado para la toma de decisiones, una modificación en el lenguaje financiero internacional y de esta forma dar un paso hacia una mejor apertura económica del país. Sin embargo, son varias las inquietudes que genera la implementación de este sistema, entre estas se encuentra la pertinencia de este sistema en el contexto colombiano. Mediante el análisis de la afectación de la implementación de este nuevo marco en el costo de capital se busca dar indicios para resolver parte estas preguntas.

En la tarea de descifrar esta inquietud; en este trabajo se determina que la aplicación de las IFRS en las principales empresas colombianas que están listadas en el índice bursátil COLCAP, no genera ningún efecto en el costo de capital, esto se puede dar debido a que la normativa anterior tenía la misma robustez en su aplicación, por tal motivo el efecto en el capital social es indiferente a la normativa contable utilizada. Así mismo se puede identificar que otras variables incluidas en el estudio si tuvieron un efecto en el costo de capital, como lo fue la inflación, el endeudamiento, el retorno sobre patrimonio (ROE), el margen operacional (MO) y el crecimiento. Sin embargo, cabe anotar que deacuerdolos trabajos revisados a nivel mundial, existela generalidad que la aplicación de las IFRS tiene efecto en la diminución del costo de capital en las empresas privadas.

Estos resultados aportan a los enfoques financieros que se encuentra en la búsqueda de nuevas variables que pueden afectar la rentabilidad de una inversión; de igual forma se presume que las IFRS aportan de igual media que la normativa anteriormente aplicada, a la reducción de la asimetría de la información y al paradigma de la utilidad de la información desde el punto de vista del capital social. Por último, se resalta que, para futuras investigaciones, se podría incluir un mayor rango de datos a analizar y adicionalmente, sería pertinente realizar este tipo de análisis para empresas que no cotizan en la bolsa de valores.

\section{Referencias bibliográficas}

Agustin,A. (2016). The effect of firm size and rate of inflation on cost of capital: The role of ifrs adoption in the world. procedia-social and behavioral sciences, 219(1), 47-54. Doi: 10.1016/j.sbspro.2016.04.031 
Laverde, M., Lezama, J., García, J., Montoya, I., Pérez, E. El efecto en el costo de capital...

American Institute of Certified Public Accountants. (2019). IFRS FAQS. Recuperado de https://www.ifrs.com/ifrs_faqs.html\#ftnt1

Bae, K., Tan, H., y Welker, M. (2008). International GAAP Differences: The Impact on Foreign Analysts. The Accounting Review, 83, 593-628.

Ball, R. (2006). IFRS Pros and Cons for investors. The Accounting Review, 2006, 593-628.

Barth, M., Landsman, W., y Lang, M. (2008). International Accounting Standards and Accounting Quality. Journal of Accounting Research, 46, 67-98. Doi:10.1111/j.1475679X.2008.00287.x

Baum, C. (2001). Residual diagnostics for cross-section time series regression models. The Stata Journal, 1(1), 101-104. Doi: 10.1177/1536867X0100100108

Belkaoui, A. (1993). Accounting theory. Reino Unido: Harcourt Brace.

Besley, S., Brigham, E., y Gomez, J. (2001). Fundamentos de Administración Financiera. México: Mc. Graw Hill.

Botosan, C. (1997). Disclosure level and the cost of equity capital. Accounting review, 1(1), 323-349.

Brealey, R., S., M., y F., A. (2010). Principios de Finanzas Corporativas. Ciudad de México: Mc Graw Hill.

Breusch, T., y Pagan, A. (1980). The Lagrange multiplier and its applications to model specificatión in econometrics. Review of Economics Studies, 47(1), 1251-1271. Doi: 10.2307/2297111.

Byard, D., Li, y., y Yu, y. (2011). The Effect of Mandatory IFRS Adoption on Financial Analysts' Information Environment. Journal of Accounting Research, 49(1), 69-96. Doi:10.1111/j.1475-679X.2010.00390.x

Castillo, D., Menédez, C., y Orgaz, N. (2014). Mandatory IFRS adoption and the cost of equity capital: Evidence from Spanish firms. Intangible Capital, 10(3), 562-583. Doi: http://dx.doi.org/10.3926/ic.491

Damodaran, A. (2002). Investment Valuation. New York: Wiley.

Damodaran, A. (n.d.). Damodaran Online. Recuperado de http://people.stern.nyu.edu/ adamodar/

Daske, H., Hail, L., Leuz, C., y Verdi, R. (2008). Mandatory IFRS Reporting around the World: Early Evidence on the Economic Consequences. Journal of Accounting Research, 46, 1085-1142. Doi: https://doi.org/10.1111/j.1475-679X.2008.00306.x

Diamond, D., y Verrecchia, R. (1991). Disclosure, liquidity, and the cost of capital. The journal of Finance, 46(4), 1325-1359. Doi: 10.2307/2328861

Easton, P. (2004). PE Ratios, PEG Ratios, and Estimating the Implied Expected Rate of Return on Equity Capital. The Accounting Review, 79(1), 73-95.

Farfán, M. (2010). Análisis de la vigencia del paradigma de utilidad en la contabilidad moderna. Lúmina, 242-263.

Foster. (2013). FASB web Site. Recuperado de https://www.fasb.org/articlesyreports/ Foster_FASBReport.pdf

Gatsios, R., Da Silva, J., Ambrozini, M., Neto, A., y Lima, F.(2016). Impact of adopting ifrs standard on the equity cost of brazilian open capital companies. RAM revista 
de administracion mackenzie, 17(4), 85-108. Doi: http://dx.doi.org/10.1590/167869712016/administracao.v17n4p84-107

Góis, A., de Lima, G., de Sousa, N., y Malacrida, M. (2018). Relationship between IFRS Adoption and the Cost of Equity Capital. Journal of International Accounting Research, 17(3), 69-85. doi:10.2308/jiar-52192

Gómez, C., y García, M. (2011). Supuestos implícitos en la utilización del Capital Assets Pricing Model-CAPM - para el cálculo del costo del capital propio - Equity. Documetos Doctorado documentos fce-cid, 1-26.

Gordon, J. (1997). Comportamiento organizacional. Nueva York: Prentice hall Hispanoamericana.

Gordon, M., y Shapiro, E. (1956). Capital equipment analysis: the required rate of profit. Management science, 3(1), 102-110.

Grabinski, K., Kedzior, M., y Krasodomska, J. (2014). The Polish accounting system and IFRS implementation process in the view of empirical research. Accounting and Management Information Systems, 13(2), 281-310.

Greene, W. (2000). Econometric Analysis. Upper Saddle River, New Jersey: Prentice Hall. Hausman, J. (1978). Specification test in econometrics. Econometrica, 46(1), 1251-1272. Hendriksen, E., y Fernandez, M. (1981). Teoría dela Contabilidad. México: Uthea.

Houqe, N., Monem, R., y Zijl, T. (2016). The economic consequences of IFRS adoption: Evidence from New Zealand. Journal of International Accounting, Auditing y Taxation, 27, 40-48. Doi: https://doi.org/10.1016/j.intaccaudtax.2016.10.001

Ionașcu, M., Ionașcu, I., Sâcârin, M., y Minu, M. (2014). IFRS adoption in developing countries: the case of Romania. Accounting and Management Information Systems, 13(2), 311-350.

Jensen, M., Black, F.,y Scholes, M. (1972). The capital asset pricing model: Some empirical tests.

Junta de Normas Internacionales de Contabilidad. (2018). Marco conceptual IFRS Conceptos Principales . Marco conceptual para la presentación de informes Financieros. Londres, Inglaterra, Reino Unido: International Financial Reporting Standards.

Kim, J., Shi, H., y Zhou, J. (2014). International Financial Reporting Standards, institutional infrastructures, and implied cost equity capital around the world. Quant Finan Acc, 469-507. Doi: https://doi.org/10.1007/s11156-013-0350-3

Lambert, R., Leuz, C., y Verrechia, R. (2007). Accounting Information, Disclosure, and the Cost of Capital. Journal of accounting research, 45(2), 385-420. Doi: https://doi. org/10.1111/j.1475-679X.2007.00238.x

Lee, E., Walker, M., y Christensen, H. (2008). Mandating IFRS: Its Impact on the Cost of Equity Capital in Europe. ACCA Research Report, 1-28.

Li, S. (2010). Does Mandatory Adoption of International Financial Reporting Standards in the European Union Reduce the Cost of Equity Capital? TheAccounting Review, 85(2), 607-636.

Lintner,J.(1965). Security prices, risk, and maximal gains from diversification. Thejournal of finance, 20(4), 587-615. Doi: 10.2307/2977249 
Laverde, M., Lezama, J., García, J., Montoya, I., Pérez, E. El efecto en el costo de capital...

Lucas, R. (1984). Money in a Theory of Finance. Carnegie-Rochester Conference Series on Public Policy, 21, 9-48.

Markowitz, H. (1952). Portfolio selection. The journal of finance, 7(1), 77-91.

Modigliani, F., y Miller, M. (1958). The cost of capital, corporation finance and the theory of investment. The American, 48(3), 261-297.

Mossin, J. (1966). Equilibrium in a capital asset market. Econometrica: Journal of the econometric society, 768-783.

Munteanu, L. (2011). Cost of equity, financial information disclosure, and IFRS adoption: a Iiterature review. Int. Audit. Risk Manage, 67-80.

Ohlson, J. A., y Juettner-Nauroth, B. (2005). Expected EPS and EPS Growth as Determinants of Value. Review of Accounting Studies, 10(2-3), 349-365.

Persakis, A., y Latridis, G. (2017). The joint effect of investor protection, IFRS and earnings quality on cost of capital: An international study. Journal of International Financial Markets, 46(1), 1-29. Doi: https://doi.org/10.1016/j.intfin.2016.10.001

Sharpe, W. (1964). Capital asset prices: A theory of market equilibrium under conditions of risk. The journal of finance, 19(3), 425-442. Doi: https://doi. org/10.1111/j.1540-6261.1964.tbo2865.x

Silva,A.,Augusto, J.,y Costa,M.(2016).Custo docapital próprioem empresas brasileiras do setor sucroenergético: um estudo considerando a adoção das normas internacionais decontabilidade. Universo Contabil, 12(2), 117-137. Doi:10.4270/ruc.2016217

Tenjo, F., López, E., y Zamudio, N. (2006). Determinantes de la estructura de capital delas empresas colombianas: 1996-2002. Coyuntura Económica, 1, 117-147.

Urquiza, F., Navarro, M., y Trombetta, M. (2012). Disclosure Strategies and Cost of Capital. Managerial and Decision Economics, 33(7), 501-509. Doi: https://doi.org/10.1002/ mde. 2562

Valderrama,M., Díez,J.,y Gaitán,S.(2011).Aproximación alas metodologías deestimación del costo de capital en los proyectos de inversión. El caso colombiano. AD-minister, 101-124.

Weston, J.(1963). Theinvestment, financing, and valuation of the corporation. The Journal of Finance, 18(3), 579-581. Doi: 10.2307/2977577

Weston, J., Brigham, E., y Mont, J. (1987). Fundamentos de administración financiera. México D.F.: Interamericana.

Wooldridge, J. (2002). Econometric Analysis of Cross Section and Panel Data. London, England: The MIT Press. 